\title{
Ultrasonographic measurement of uveal melanoma thickness: interobserver variability
}

\author{
Devron H Char, Stewart Kroll, Robert D Stone, Roger Harrie, Barry Kerman
}

\begin{abstract}
We retrospectively reviewed ultrasonographic data on 32 uveal melanomas that had been studied both at a referral institution and at our centre within a six-week period. Generally there were good correlations between ultrasonographic measurements of tumour thickness. The mean absolute difference in ultrasound measurements of tumour thickness was $0.64 \mathrm{~mm}$ (range $0-2.2 \mathrm{~mm}$ ); the mean tumour thickness was $5 \cdot 2 \mathrm{~mm}$ (range 3.5-10.2 $\mathbf{m m}$ ). Tumour diameter, location, the relative ultrasonographic experience with uveal melanoma, type of equipment, and tumour thickness all affected the interobserver variation of measurement.
\end{abstract}

Measurement of uveal melanoma thickness is important in the choice of therapy, prediction of radiation complications, and monitoring the efficacy of treatment. ${ }^{1}$ We and others have previously studied the accuracy of ultrasonographic measurement of uveal melanoma elevation in a single institution. ${ }^{2-4} \mathrm{We}$ observed that multiple measurements of the same tumour in the posterior pole had a variation of less than 0.5 $\mathrm{mm}$, and there was an excellent correlation between ultrasonographic and clinical estimation of tumour height. ${ }^{2}$ The accuracy of ultrasonographic measurements of tumour thickness of the same tumour in different centres is unknown. This issue is germane to the conduct of multicentre trials of uveal melanoma treatment. Tumour elevation is one of several measurements used in the choice of treatment, and it has been shown to be an important prognostic parameter for both radiation complications and survival. ${ }^{56}$

The interobserver variability of tumour measurements with the same or different ultrasound instruments is uncertain. We have reviewed ultrasonographic data on 32 uveal melanomas that had ultrasonography performed by both the referring institution and us within a six-week period. We observed that tumour thickness and diameter, the experience of the ultrasonographer, the type of equipment, and anterior location of tumour all affected the interobserver variation in the measurement of tumour thickness.

\section{Materials and methods}

We retrospectively reviewed all charts of patients with uveal melanoma who had been referred to one of us (DHC) for therapy. Those cases that had been referred with photographic documentation of ultrasonographic studies were selected for further investigation. Criteria for inclusion in this analysis were uveal melanomas not involving the iris that had complete clinical, photographic, ultrasonographic (immersion B scan and Kretz standarised A scan) data from our institution, and ultrasonographic data that had been performed within six weeks prior to our examination from the referring institution.

All ultrasonographic studies performed at our institution were done with both immersion B scan (Sonometrics model 150, New York) and standardised A scan (7200 MA Kretztechnique, Vienna, Austria). The scanning techniques used with these two instruments have previously been published. ${ }^{78}$ All patients were first studied with a $10 \mathrm{mHz}$ focused $\mathrm{B}$ scan probe to find the maximum tumour thickness, and then had a standardised A scan performed with an unfocused $8 \mathrm{mHz}$ transducer.

All outside scans were performed by a single individual in each centre with experience in ultrasonography. In 16 cases Kretz standardised A scans had been done at the referring institution, and those ultrasonographers had participated in a training course at the University of Iowa. Seven cases were studied with standardised Biophysic A scan. Nine patients were referred from the University of California at Los Angeles, seven from Denver, three from Salt Lake City, three from the University of Southern California, and one each from a number of other centres. Each centre was poled to determine the number of uveal melanomas studied by ultrasound each year in that centre.

We reanalysed all ultrasound data, and the standardised A scan measurement of tumour thickness was defined as the distance between the anterior tumour spike and the anterior scleral spike in microseconds multiplied by 0.766 to obtain tumour thickness in millimetres. This value was chosen as a composite velocity factor for the entire intraocular path of an ultrasound beam. If the anterior tumour spike could not be visualised, the anterior retinal surface spike was used. As a separate control in all cases that had both $A$ and $B$ scans performed these data were separately analysed for measurement inconsistencies; no case showed a marked variation between these two techniques that would have suggested a sampling error. In cases that did not have standardised A scan performed at outside institutions the B scan measurements, usually digitally displayed, were used for tumour thickness, and compared with the non-standardised A scan data. B scan data were analysed with a velocity of both $1532 \mathrm{~m} / \mathrm{s}$ and $1550 \mathrm{~m} / \mathrm{s}$.

All patients in this investigation were studied prior to either ${ }^{125}$ I brachytherapy or charged particle (helium ion) irradiation. Statistical analysis was performed by both univariate and multivariate analysis. The covariates used in the analysis included tumour location (distance from nerve, fovea, and relationship between the

\footnotetext{
Correspondence to: D H Char, MD, Ocular Oncology Unit, Francisco, CA 94143, USA Accepted for publication 5 October 1989

University of California, Los Angeles, Jules Stein Eye Institute
} 
anterior edge of the melanoma and the equator of the eye), clinical tumour diameter, tumour thickness (measured by ultrasound), type of ultrasound equipment used, the institution where ultrasonography was performed, and interval between ultrasonographic examinations.

\section{Results}

All patients were studied prior to radiation therapy. Eighteen patients received helium ion irradiation and 14 received ${ }^{125} \mathrm{I}$ brachytherapy. Ultrasonographic tumour characteristics are summarised in Table $I$. The mean tumour thickness, measured with Kretz A scan in our laboratory, was $5 \cdot 2 \mathrm{~mm}$ (range $3 \cdot 5-10 \cdot 2 \mathrm{~mm}$ ). The average of the ultrasound tumour thickness measurements performed at the University of California, San Francisco, was $\mathbf{0 . 2 5} \mathrm{mm}$ larger than those from other institutions. The mean absolute difference in ultrasound measurements of thickness was $0.64 \mathrm{~mm}$ between the referring unit and our unit (Fig 1; range 0-2.2 mm; sd 0.60 $\mathrm{mm})$. As shown in Figure 1, large differences in ultrasonographic measurements were observed in both large and small tumours, though there was a trend towards larger differences in larger tumours. The average time between measurements was three weeks, with a maximum of six weeks.

Twenty-three tumours were classified as medium size melanomas $(3-5 \mathrm{~mm}$ in thickness and $10-15 \mathrm{~mm}$ in diameter). Nine patients had large melanomas $(>5 \mathrm{~mm}$ in thickness and/or $>15 \mathrm{~mm}$ in diameter); six melanomas were over $15 \mathrm{~mm}$ in greatest diameter. Tumour locations in the eye are shown in Table I. Fifteen patients had tumours confined to the posterior pole. Seven patients had tumours that were entirely anterior to the equator, and 10 patients had tumours that involved the choroid both anteriorly and posteriorly to the equator. Two tumours involved the ciliary body. No iris melanomas were included in this series.

The correlation between the ultrasound measurements from referring institutions and our own was $\mathbf{0} \cdot 89$. A number of factors affected these correlations. There were greater differences in ultrasound measurements from centres that examined fewer ( $<20$ per year) versus more uveal melanomas $(0.80 \mathrm{~mm}$ versus $0.40 \mathrm{~mm})$, tumours with the anterior edge anterior to the equator $(0.85 \mathrm{~mm}$ versus $0.47 \mathrm{~mm})$, institutions that did not use Kretz standardised A scan techniques $(0.50 \mathrm{~mm}$ versus $0.80 \mathrm{~mm})$, and studies performed on progressively larger tumours $(1.00 \mathrm{~mm}$ versus $0.50 \mathrm{~mm})$. The correlation between the variations in ultrasound measurement of tumour thickness and increased tumour height were significant $(r=0.524$;

TABLE I Uveal melanoma location

\begin{tabular}{lc}
\hline Tumour characteristics & \\
\hline Location & Number of \\
\hline Pomours \\
\hline Posterior to equator & 15 \\
$<3 \mathrm{~mm}$ nerve and fovea & 7 \\
$\geqslant 3 \mathrm{~mm}$ nerve or fovea & 8 \\
Involving both anterior and posterior choroid & 10 \\
Anterior to equator, not involving ciliary body & 5 \\
Involving ciliary body & 2 \\
\hline
\end{tabular}

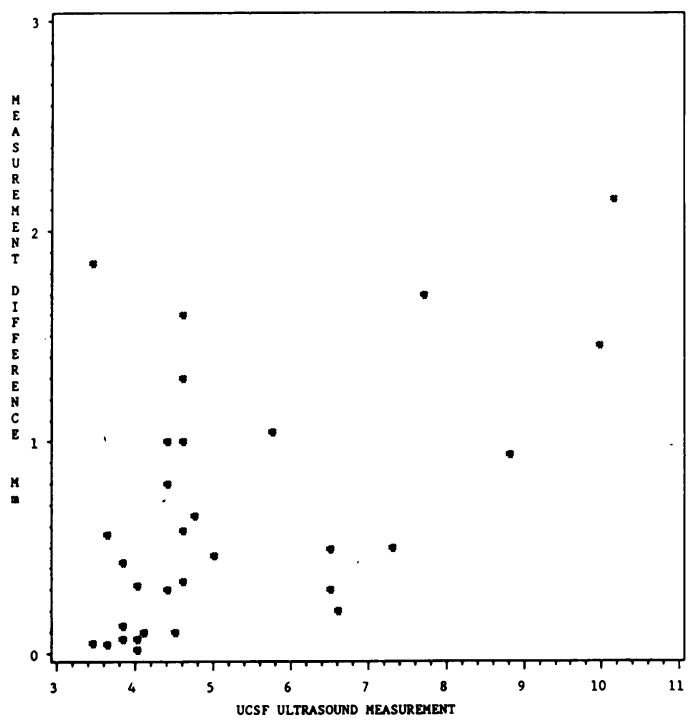

Figure 1: Range of absolute ultrasound differences in tumour thickness measurements between different institutions. (UCSF=University of California, San Francisco.)

$\mathrm{p}<0.002$ ) (Fig 1). Thicker tumours were more likely to have a greater difference in ultrasonographic measurements than thinner tumours. A low level of correlation was noted between the time interval between the two ultrasound studies and the difference of thickness measurements (Fig 2).

We reanalysed the five cases that had the largest discrepancies $(>1.3 \mathrm{~mm})$ between the referring unit's and our ultrasound measurements. All were tumours over $11 \mathrm{~mm}$ in diameter and at least partially involved the area anterior to the equator. Three extended anterior to the ora serrata, and two had clinically obvious breaks in Bruch's membrane. All of these cases were referred from centres that examine fewer than 10 melanomas a year, and only one had been studied with standardised A scan.

We studied mathematical models to investigate factors that lead to variations in the absolute differences between interinstitution ultrasound measurements. A general linear model was fitted with the ultrasound absolute differences as the dependent variables. Several data points with the

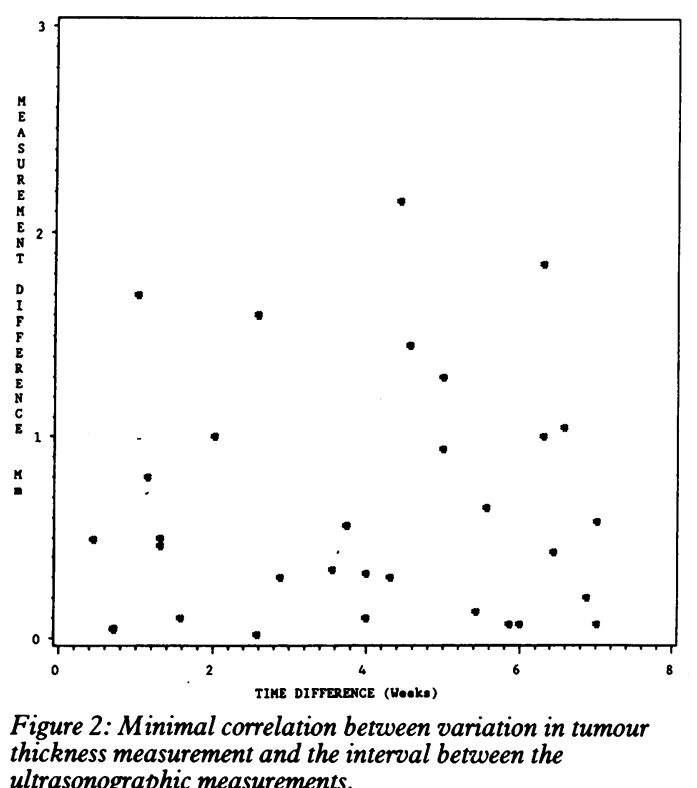


TABLE II Ultrasonographic tumour characteristics

\begin{tabular}{|c|c|c|c|c|}
\hline \multirow[b]{2}{*}{ Variable } & \multicolumn{4}{|c|}{ Ultrasonographic characteristics } \\
\hline & Minimum & Maximum & $S D$ & Mean \\
\hline Largest tumour diameter (mm) & $5 \cdot 5$ & $16 \cdot 0$ & $3 \cdot 1$ & $11 \cdot 1$ \\
\hline Ultrasound height $\left(\mathrm{UCSF}^{\star}\right)$ & $3 \cdot 45$ & $10 \cdot 15$ & 1.84 & $5 \cdot 17$ \\
\hline Ultrasound height (other centres) & $3 \cdot 00$ & $8 \cdot 50$ & $1 \cdot 49$ & $4 \cdot 91$ \\
\hline Difference in ultrasound height & -1.85 & $2 \cdot 15$ & 0.85 & $0 \cdot 25$ \\
\hline Absolute differences & 0.02 & $2 \cdot 15$ & 0.60 & 0.64 \\
\hline Time between measures (months) & 0.01 & $1 \cdot 41$ & $0 \cdot 50$ & 0.91 \\
\hline
\end{tabular}

«University of California, San Francisco.

largest differences were overly influential, and when these data points were excluded the model changed significantly. This effect was minimised, and the observations were symmetrised, when the square roots of the absolute differences were used as the dependent variables. Using the general linear model with covariates listed in the 'Methods' section resulted in a best subsets model as follows (standard errors for the estimates are given in parentheses):

$$
\begin{aligned}
& \sqrt{\text { Differences }}= \\
& 0.085 \text { (ultrasound) }+0.024 \text { (diameter) }-0.21 \text { (number of cases) } \\
& (0.032) \\
& (0.016)
\end{aligned}
$$

Ultrasound equals the ultrasound measurement taken at our oncology unit, diameter equals the tumour's largest diameter, and number of cases is an indicator variable for relative melanoma experience at referring centres. None of the other covariates had $t$ statistics larger than 0.80 except the time between the measurements, which had an associated estimate of 0.12 with a standard error of $0 \cdot 11$. The mean square error was $0 \cdot 102$. The model was selected in an exploratory fashion with numerous possible covariates, and the model may therefore not be generally applicable. These data suggest that larger differences in ultrasonographic measurements of tumour thickness between centres can be expected in thicker tumours, those with larger diameters, and when ultrasonography is performed in centres that examine relatively few melanomas a year. In this data set the location of the tumour was confounded with tumour size and centre experience, so that a specific parameter was not separately analysed in our model. This mathematical model serves two purposes. One, the parameter estimates suggest the degree ultrasonographic measurements vary as the covariates change. Two, it is a first approximation to estimate expected measurement differences for observations having specific covariates.

\section{Discussion}

A number of factors influence the accuracy of ultrasound measurements of uveal melanomas. Several publications have addressed some of these issues when ultrasonography is performed in a single institution. ${ }^{2-4}$ In most cases the variation is less than $0.5 \mathrm{~mm}$ in melanomas confined to the posterior pole. However, measurement discrepancies may increase in anterior lesions, those with an uneven surface topography (especially if an isolated break in Bruch's membrane is present), or if shifting subretinal fluid is present over the tumour. ${ }^{2}$ For thicker tumours with a steep rise that are measured with standardised A scan it is essential to have the probe perpendicular to the tumour surface, since measurements obtained even slightly off axis result in errors. Even when a B scan is done before A scan measurements, in larger lesions it is still possible to fail to sample the thickest part of the melanoma, especially if there is only a small off-centre or multiple asymmetric breaks in Bruch's membrane.

Technical factors may also lead to measurement errors. ${ }^{9}$ In this study we noted that the mean difference between measurements was approximately $0.7 \mathrm{~mm}$; the mean difference decreased to approximately $0.5 \mathrm{~mm}$ in smaller posterior melanomas, especially when measured by experienced observers with standardised A scan. The degree of difference between institutions was less than we expected. In a single institution Nicholson and coworkers noted that, when two ultrasonography technicians from the same laboratory independently measured previously performed scans, approximately $90 \%$ were within $0.4 \mathrm{~mm}$ of each other with a standard deviation $0.2 \mathrm{~mm}^{4}{ }^{4}$ These data suggest that attempts further to decrease the interobserver, interinstitution variation would be unlikely to be successful.

Peyster and colleagues studied the accuracy of ultrasound and computed tomography (CT) in the measurement of tumour thickness, and their finding may have relevance, especially for multicentre trials. ${ }^{10}$ They noted a close correlation between these two measures of tumour thickness $(\mathrm{r}=0.92)$. An obvious advantage of CT studies is that they are automated, unlike ultrasound, and therefore the measurement of tumour thickness is more likely to be consistent even in less experienced centres. While in general there was good correlation between centres in our study, some marked differences were noted. In one case the two measurements varied by over $2 \cdot 0 \mathrm{~mm}$. Since these larger differences could lead to inaccurately planned radiation therapy, it is imperative to restudy all cases prior to treatment, and if a marked difference is noted between the clinical and ultrasonographic measurement, to confirm the tumour size with either computed tomographic or magnetic resonance imaging.

This work was supported in part by NIH grant EYO 7504 American Cancer Society Grant PDT-321, and unrestricted grants from That Man May See, the Beal Foundation, Marvin Mintz, and the Weltkunst Foundation.

1 Char DH. Clinical ocular oncology. New York: Churchill Livingstone, 1989.

2 Char DH, Stone RD, Irvine AR, et al. Diagnostic modalities in choroidal melanoma. Am f Ophthalmol 1980; 89: 223-30.

3 Ossoinig DC, Bigar F, Kaefring SL. Malignant melanoma of the choroid and ciliary body. A differential diagnosis in clinical echography. Bibl Ophthalmol 1975; 83: 141 .

4 Nicholson DH, Frazier-Byrne S, Chiu MT, Schiffman J, Hughes JR, Novinski EK. Echographic and histologic tumor height measurements in uveal melanoma. $A m \mathcal{F}$ Ophthalmol 1985; 100: 454-7.

5 Seddon JM, Gragoudas ES, Polivogianis L, et al. Visua outcome after proton beam irradiation of uveal melanoma. outcome after proton beam irradiat
Ophthalmology 1986; 93: 666-74.

6 Kindy-Degnan NA, Char DH, Castro JR, et al. Effect of various doses of radiation for uveal melanoma on regression visual acuity, complications and survival. Am $\mathcal{f}$ Ophthalmol 1989; 107: 114-20.

7 Ossoinig K. Echography of the eye, orbit, and periorbital region. In: Arger PH, ed. Orbit roentgenology. New York: Wiley, 1977: 224

8 Coleman DJ, Lizzi FL, Jack RL. Ultrasonography of the eye and orbit. Philadelphia: Lea and Febiger, 1977.

9 Coleman DJ. Echographic histologic tumor height measurements in uveal melanoma. Am $\mathcal{J}$ Ophthalmol 1986; 101 $124-5$

10 Peyster RG, Augsburger JJ, Shields JA, et al. Choroida melanoma: comparison of CT, fundoscopy, and US Radiology 1985; 156: 675-80. 